\title{
BMJ Open Understanding and scaffolding Danish schoolteachers' motivation for using classroom-based physical activity: study protocol for a mixed methods study
}

\author{
Louise Stjerne Knudsen, ${ }^{1,2}$ Thomas Skovgaard, ${ }^{1,2}$ Thomas Bredahl $^{1}$
}

To cite: Knudsen LS, Skovgaard T, Bredahl T. Understanding and scaffolding Danish schoolteachers' motivation for using classroombased physical activity: study protocol for a mixed methods study. BMJ Open 2018;8:e019857. doi:10.1136/ bmjopen-2017-019857

- Prepublication history and additional material for this paper are available online. To view these files, please visit the journal online (http://dx.doi org/10.1136/bmjopen-2017019857).

Received 29 September 2017 Revised 4 February 2018 Accepted 13 February 2018

\section{Check for updates}

1Department of Sports Science and Clinical Biomechanics, University of Southern Denmark and Research, Odense, Denmark ${ }^{2}$ Department of Sports Science and Clinical Biomechanics, Research and Innovation Centre for Human Movement and Learning, Odense, Denmark

Correspondence to Ms Louise Stjerne Knudsen; Isknudsen@health.sdu.dk

\section{ABSTRACT}

Introduction The benefits of physical activity for children's health, both mental and physical, and its positive effects on academic achievement are well established.

Research also emphasises that schools could provide a natural setting for regular physical activity. There is, however, a limited amount of knowledge about teachers' views when it comes to integrating physical activity as part of teaching. The aim of this study is to understand teachers' motivation for integrating physical activity as part of teaching and to assess their need for guidance and support.

Methods and analysis The study uses an explanatory sequential mixed-methods design. Schools from across Denmark are included in the sample. The design comprises two separated phases-a quantitative and qualitative phase. The quantitative phase is guided by the self-determination theory where teachers' motivation will be measured using the Work Task Motivation Scale for Teachers. The theory of scaffolding guides the qualitative phase, which consists of in-depth interviews with participants selected from the quantitative phase based on levels of motivation and on demographic information. In accordance with the study aims, the analysis of data will identify teachers' internal and external levels of motivation. The purpose of the qualitative phase is to enhance understanding of teachers' motivation and of their need for support in the use of physical activity in teaching.

Ethics and dissemination All relevant ethics approvals have been acquired. All participants in this study will provide written informed consent prior to data collection. All data emerging from the quantitative and qualitative phase will be anonymised for analysis. Ethics approval was requested from the Regional Committee on Health Research Ethics for Southern Denmark approval ID S-20162000-40 and the Danish Data Protection Agency approval ID 16/15491). The study was deemed not notifiable by both authorities.

Trial registration number NCT02894346; Pre-results.

\section{BACKGROUND AND INTRODUCTION}

The benefits of physical activity (PA) for mental and physical health, cognitive function and academic achievement are well established, particularly for children and young people. ${ }^{1-6}$ Children and adolescents

\section{Strengths and limitations of this study}

- The mixed methods approach provides a nuanced and detailed understanding of teachers' motivation and need of scaffolding.

- The study involves a broad selection of Danish schoolteachers across age, gender, experience and school type.

- The study uses a systematic recruiting strategy making sure that different levels of motivation are represented.

- The study has the potential to address key factors of future school-based interventions that seek to increase students' levels of physical activity during the school day.

- The study covers a wide range of areas of Denmark but will not be representative for all teachers in Denmark.

spend a large part of the day at school, and even though they are required to sit still for the majority of the day, research emphasises that school is an ideal setting for promoting PA. ${ }^{78}$ In Denmark, daily PA has been mandatory in all Danish state schools (primary and lower secondary education) since 2014 and must be integrated throughout the school day. Physical education has been an integrated part of the curriculum in Danish state schools for many years; however, daily PA is part of a comprehensive public school state reform launched in August 2014. The purpose is to ensure that all students engage in sport, exercise and PA everyday with the purpose of securing their health and wellbeing and at the same time supporting motivation and learning in all subjects. In this study, PA is used in accordance with the terms in the reform text, which is exercise, sport and movement and offers a broad understanding of movement activities. All students must engage in, on average, $45 \mathrm{~min}$ of $\mathrm{PA}$ during the school day. PA has to be part of subject-divided teaching (eg, mathematics, 
science, language subjects, history, geography, physical education, etc) and of assisted learning. This means that all subject teachers across the curriculum are responsible for using PA. Assisted learning supplements and supports subject-divided teaching and is typically placed at the end of the school day. ${ }^{9}$ In this study, 'teacher' is an overall label for both teachers and assistant teachers. Assistant teachers are often part of both subject-divided teaching and assisted learning with the purpose of supporting the main teacher and also carry out assisted learning alone. School management has to ensure that the physically active school day is, in fact, implemented. However, there are no recognised guidelines from the Danish Ministry of Education in this area nor are there any consequences if the school does not adhere to the PA policy. Danish schoolteachers do not, in general, receive training in facilitating physical activities in the classroom during their formal teacher education. However, teachers who choose physical education as main subject during their education, receive training in developing, for instance, PAs. At the moment, selected national school-sport organisations offer professional development for teachers focusing on the PA policy. However, these training courses are not mandatory.

A few preliminary Danish reports on the implementation of the state school reform have been conducted..$^{10} 11$ These state that a considerable number of schools and teachers find it difficult to integrate PA into the school day. The reports note that PA has a positive effect on students' well-being and academic performance, but that barriers to integrating PA during the school day remain and that only a limited number of schools have successfully implemented PA. ${ }^{10}{ }^{11}$ There is an implication that teachers need more guidance and support in integrating $\mathrm{PA}$ in a meaningful manner, especially as part of their teaching practice. ${ }^{10} 11$ However, there are no suggestions as to what kind of support or who should provide it. As a result, we know very little of the effect of the reform and teachers' qualifications and motivation for integrating PA.

As far as we can see, there is a shortage of knowledge about teachers' motivation for integrating PA into the classroom. In this sense, 'integration' means incorporating PA into the academic material of any given subject. Looking at the literature, we find suggestions of the perceived barriers to integrating PA into the classroom, ${ }^{12-14}$ and these are typically time consumption, other curriculum pressures, lack of resources, lack of space and lack of competence. Additionally, a number of studies address the issue of teachers' perspectives on $\mathrm{PA}^{13} 15-18$ and willingness to integrate PA into the classroom. ${ }^{19}{ }^{20}$ However, knowledge about the factors that may influence teachers' motivation or view on classroom-based PA is limited. A study by Parks $e t a l^{19}$ found that teachers generally recognise the importance of PA for children's physical and mental health and possibly for their academic progress. The study focused on elementary schoolteachers and school principals recruited from both public and private schools in the USA. Elementary school in the USA corresponds to preschool class to fifth grade in Denmark (6-11 years of age). The findings of the study also indicates that teachers would be willing to integrate PA into the classroom but that their preparedness for designing and implementing PA is limited. It finds that they would need support to do so successfully. The Parks et al study raises important concerns about teachers' motivation for integrating PA into the classroom and about the factors that may hinder or facilitate that integration. The study also supports the notion that integrating something 'new', such as school-based PA interventions or a state school reform such as the one seen in Denmark, puts extra pressure on teachers that affects their involvement in the particular new task and in their overall job satisfaction. ${ }^{21} 22$ Furthermore, since teachers' motivation has received little attention compared with students' motivation, ${ }^{23-27}$ it is very relevant to understand their motivation in an educational setting. The reform introduced in Denmark is rather unique, in the way that it is mandatory as opposed to guided by broadly based recommendations about PA in schools (eg, the United States: Comprehensive School Physical Activity Program). All state schools in Denmark are obligated to integrate $45 \mathrm{~min}$ of PA during the school day across year groups and subjects. Teachers therefore play an important role in integrating PA into the classroom and in increasing levels of PA among students. ${ }^{28}{ }^{29}$ In the light of the reform, it is therefore even more necessary to understand teachers' motivation for the new PA task, as well as their need for support in integrating PA into the classroom. ${ }^{17}$ This study investigates teachers' motivation and need for support across subjects and year groups using two underpinning theories as a guide.

\section{Self-determination theory}

In this project, the self-determination theory (SDT) ${ }^{30} 31$ is applied and motivation assessed using the Work Task Motivation Scale for Teachers (WTMST) ${ }^{32}$ SDT is a useful theoretical framework for understanding the nature of teachers' motivation. SDT makes important distinctions between different types of motivation that make human beings act in different domains. Using SDT will make it possible to establish whether teachers have the motivation to use PA as part of their teaching on a daily basis. Furthermore, by applying the SDT perspective, this study will look further into possibilities of maintaining PA in both subject-divided teaching and assisted learning.

At the core of SDT are three basic psychological needs that, according to Deci and Ryan, are inherent in all human life and essential for optimal human functioning and development. The three innate needs are competence, relatedness and autonomy. Competence can be described as a feeling of being able to achieve a possible and/or desired outcome. Relatedness is the feeling of connectedness with others and having a sense of belonging. Autonomy refers to the feeling of self-determination. ${ }^{30} 313334$ Deci and Ryan distinguish between 
intrinsic motivation, external regulation (external, introjected, identified and integrated) and amotivation. For example, intrinsic motivation occurs when people engage in their job for the pleasure and satisfaction they gain from it. External regulation occurs when the underlying motives are more instrumental and when reward is seen as originating in a benefit or pressure from without. ${ }^{31} 35$ There are four kinds of external regulation according to Deci and Ryan. External regulation is a classical type of extrinsic motivation. It occurs when a task is performed on the basis of rewards or to avoid punishment. Introjected regulation occurs when the work tasks are performed to avoid feelings of guilt or to increase self-worth. Identified regulation occurs when work tasks are performed based on personal beliefs, importance or personal values. According to Deci and Ryan this is the process where people recognise and accept the underlying value of a behaviour or task. ${ }^{30}$ Integration occurs when people identify with the importance of the task and integrate the identification with other aspects of the self. ${ }^{30}$ In this study, these different types of motivation and regulations will be assessed at a task level by applying the WTMST. ${ }^{32}$ For example, to what extent do teachers feel regulated by external pressure or to what extent are they intrinsically motivated when it comes to integrating PA?

\section{Scaffolding}

According to Deci and Ryan all human motivation needs supportive conditions to thrive. Negative conditions can, on the contrary, diminish motivation. The mentioned authors see the environment as a key predictor in the maintenance and support of motivation. ${ }^{30} 31$ To understand how teachers and their motivation can be supported in the process of integrating PA in teaching, scaffolding will be used as a theoretical framework. In this study, the environment will be understood as the school context, consisting of both social, cultural and physical elements. ${ }^{3637}$

Scaffolding is originally a teaching strategy and originated as the principle of an expert (eg, teacher) that guides a learner (eg, student) in a one-to-one interaction. Building on Lev Vygotsky's zone of proximal development, the basic feature of scaffolding is to guide and support children or students to solve problems not by telling them what to do but by using six types of scaffolding, which, applied appropriately, help the learner in the development process until they are able to solve the task themselves. ${ }^{38}$ The zone of proximal development is characterised as the distance between a child's actual development level and the level of potential development. ${ }^{3639}$

In this study, scaffolding will not be used as a teaching strategy in its original form. Instead it will be used as a way of describing and identifying the kind of supportive conditions that exist in a school environment. Scaffolding is therefore used synonymously with support, ${ }^{37}$ and will be used to understand what kind of support and scaffolding 'tools' teachers need to carry out PA. It will for example be possible to identify, through the six levels of scaffolding (recruitment, reduction of degrees of freedom, direction maintenance, marking critical features, frustration control and demonstration), what kind of scaffolding strategy is needed. ${ }^{38}$ Additionally, through the six levels of scaffolding means (feeding back, hints, instructing, explaining, modelling and questioning), it will be possible to further elaborate this strategy and understand what kind of assistance and support teachers specifically need. ${ }^{40}$

\section{Study aims}

This study aims to:

1. Understand teachers' motivation for using PA in teaching using SDT as a theoretical framework;

2. Understand how teachers and their motivation can be maintained and supported by using the theory of scaffolding.

\section{METHODS AND ANALYSIS}

To understand teachers' motivation, this study uses an explanatory sequential mixed methods design. ${ }^{41-43}$ The purpose is to explore preliminary quantitative data in a subsequent supplementary qualitative phase. ${ }^{44}$ The combination of quantitative and qualitative methods will provide a rich and detailed understanding of the study aims. The study started January 2016 and will end December 2018.

\section{The quantitative phase \\ Selection of sample}

The target population in this study are teachers employed at Danish state schools. Inclusion criteria are (1) employment at a Danish state school and (2) teaching responsibilities (both subject-divided teaching and assisted learning). A pilot study was conducted as part of the quantitative data collection phase. During the pilot study, it became clear that teaching assistants with special teaching tasks also carry out PA activities in assisted learning. As a result, teaching assistants were included in the sample. For the quantitative phase, a basic probability sampling technique was used. ${ }^{45}$ In the first stage of sampling, a cluster of schools was randomly selected. To secure maximum variation and diversity in the sample, schools were chosen on the basis of their regional and municipal location to reach a variety of schools to make sure that both countryside, suburban and city schools were included. By using this type of sampling technique, it was also possible to include schools that show different usage levels of PA. The final step of the sampling process was identifying the teachers at the sampled schools.

\section{Recruitment}

Participants were recruited through school management. Each school manager received a recruitment email consisting of a detailed description of the study, its purpose, time consumption and possible workload. After approval of the study at management level, teachers at 


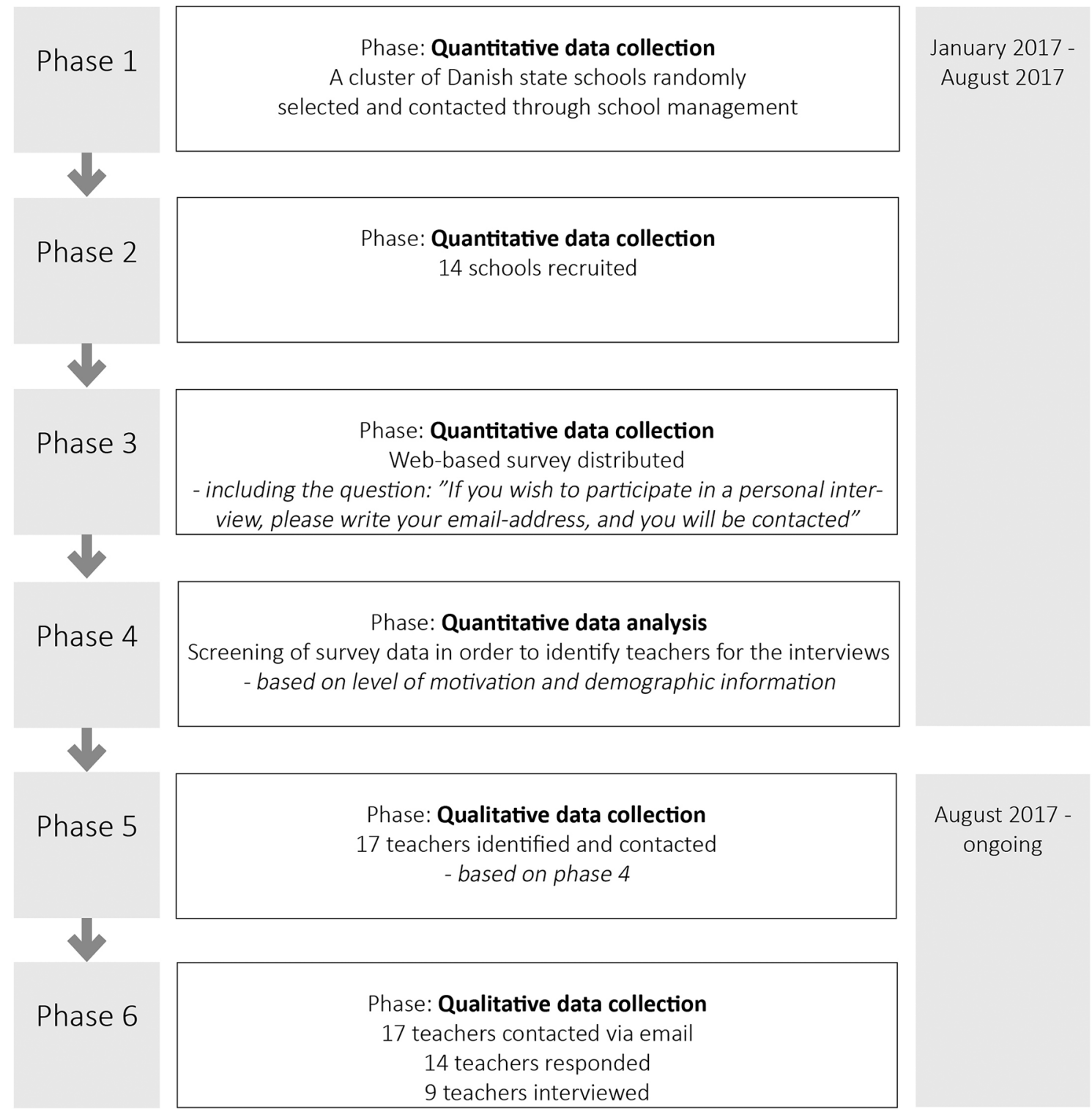

Figure 1 Overview of recruitment procedures in the quantitative and qualitative data collection phases.

each sampled school received a message through the internal school message system. The message entailed a link to the questionnaire and provided schools and participants with knowledge of handling of data, data security, participation rights and anonymity (figure 1 ).

\section{Questionnaire translation and pilot study}

A systematic adaptation, pilot testing and back-translation process of WTMST were conducted. ${ }^{46-48}$ The scale was translated from English to Danish using a bilingual translator. Next, it was culturally adapted, making sure the questionnaire was understandable and meaningful to the sample. The scale was pilot tested on teachers employed at a state school representative of the sample. After the pilot test, the scale was analysed and evaluated by a qualified and appropriate reference group. The reference group consisted of researchers in the field of physical activity, well-being and learning within school contexts. On the basis of the feedback from the reference group, further adaptions were made. The questionnaire was then back-translated into English. An expert committee consisting of one native English speaker as well as researchers with specific knowledge of teaching and the Danish school system evaluated the back-translated questionnaire. Meaning variations were discussed. No significant differences in meaning between the two English versions were identified. An original English version of WTMST can be found in online supplementary appendix 1 .

\section{Quantitative data collection}

The target group answered a questionnaire based on WTMST. The scale is designed to measure motivation towards six tasks: class preparation, teaching, evaluation of studies, classroom management, administrative tasks and complementary tasks. ${ }^{32} 35$ At the core of the questionnaire are 15 items specifically designed to measure both internal and external types of motivation, such as: 
intrinsic, identified, introjected and external regulations and amotivation. Each motivational type contains three items addressing possible reasons for engaging in a particular task. In this study, questions fall into various types, such as: 'Because I find movement activities interesting to use' (intrinsic); 'Because I feel guilty if I don't use movement activities' (introjected); 'Because I'm paid to use movement activities' (external) and 'Because it is important for me to use movement activities' (identified). The 15 items are scored on a 7-point Likert scale. WTMST will be used to measure teachers' motivation for using PA as part of their teaching task (both subject divided and assisted learning) on a task level. In addition, the questionnaire consists of questions that measure factors that either hinder or facilitate the integration of PA in a school setting. These factors are based on findings from a review in which Naylor and colleagues ${ }^{1}$ identified several perceived barriers that may influence the implementation of school-based PA interventions. Five factors were chosen for this questionnaire: time, support, resources, own competence and relevance. Participants were asked to prioritise the five factors from 1 to 5 five in two categories: (1) seen as important for carrying out PA and(2) acting as a barrier for carrying out PA. In addition, the participants answered sociodemographic questions concerning gender, age, employment status, subject(s), year(s), school and experience.

\section{Data analysis}

All data from the questionnaire will be analysed using SPSS statistical software V.24. Data from the questionnaire will be organised and summarised in a descriptive analysis for the purpose of enhancing understanding of teachers' level of motivation. ${ }^{49}$ In the descriptive analysis questionnaire, information on levels of motivation will be analysed alongside demographic information using frequency counts and cross-tabulations. This type of analysis will provide the information needed for the qualitative phase of the study.

\section{The qualitative phase}

\section{Sampling and case selection}

Participants for the qualitative phase will be selected based on the following variables: (1) their level of motivation measured by WTMST-ensuring that both intrinsic and extrinsic levels are represented- and (2) sociodemographic factors such as age, gender, subject and length of employment. The number of participants for the interviews is based on data saturation and will be ongoing until reaching the point where the data are rich and detailed and no new information arises. Recruitment of participants is an integrated part of the survey process, where respondents are asked to write their email address if they are interested in participating in a subsequent in-depth interview.

\section{Data collection}

Against the background of the preliminary quantitative findings, the aim of the qualitative phase is to elaborate on and enhance the understanding of teachers' motivation and of their need for support. ${ }^{44}$ Where the quantitative data will provide a general picture of trends based on the sample of teachers measured by the WTMST, the qualitative phase will deepen the understanding of their motivational levels based on the three psychological needs highlighted in SDT (competence, relatedness and autonomy). The qualitative phase will also explore teachers' need for support. ${ }^{41} 50$ Data will be collected through individual in-depth interviews, ${ }^{51}$ and interviews will be carried out in the participants natural setting (schools).$^{50} \mathrm{~A}$ semistructured interview guide will form the basis for the interviews, which will be conducted face to face. ${ }^{51}$ In the qualitative data collection period, we will consider teachers' teaching responsibilities and workload, thus staying clear of, for instance, examination periods. Interviews are expected to last approximately 1 hour.

The semistructured interview guide is primarily informed by the SDT and the theory of scaffolding. Participants will first be asked to identify motivational levels in accordance to the three basic needs from SDT (competence, relatedness and autonomy). Second, participants will be asked to identify the types of scaffolding levels they currently meet at a school level. Third, participants will be asked to identify what kind of support they need to carry out PA in teaching. Lastly, participants will be asked to identify the factors that may hinder or facilitate their motivation for integrating PA. Before starting data collection, the interview protocol will be pilot tested on one or two participants selected from the list of participants, whereupon it will be revised and adjusted as needed.

\section{Data analysis}

The interviews will be transcribed verbatim. Data will then be analysed through thematic coding using Nvivo software V.11. ${ }^{52}$ The first step in the data analysis is to conduct a preliminary exploration of the data by reading through the transcripts and any notes taken during the interview sessions. The second step is to code the data. This will allow for a thematic analysis on the basis of SDT, while the theory of scaffolding will be used for identifying, analysing and reporting on possible patterns concerning need for support and guidance-both at a case level and across cases. ${ }^{4153}$ As an overall guide, the qualitative data analysis follows the six steps of thematic analysis. ${ }^{52}$ A systematic double-coding process with a second analyst will be conducted in Nvivo to secure trustworthiness and test for reliability.

\section{Discussion of possible outcomes}

This study will provide a detailed understanding of teachers' motivation for using PA as part of their teaching practice. The mixed methods design makes it possible to achieve a theory-driven perspective on teachers' motivation based on $\mathrm{SDT}^{31}$ measured by the WTMST. Moreover, using the theory of scaffolding, ${ }^{38}$ the study provides information of how teachers can be supported in their work with integrating PA and what factors may affect 
their willingness to do so. The outcomes of this study may inform training programmes aiming to improve the skills of current and future teachers as regards implementation of PA into the classroom. The study also has the potential to address key factors of importance for designing future school-based interventions aiming to increase students' level of PA by providing knowledge of how to foster teachers' motivation. Finally, this study may be used to inform stakeholders, such as school management, board of governors and subject advisors on how to foster teachers' motivation for integrating PA into the classroom.

\section{ETHICS AND DISSEMINATION}

All participants in this study will receive information regarding their participation. This information will outline the study, their voluntary participation and the handling of data. Furthermore, participants have the right to withdraw from the study at any time. Prior to the quantitative phase, school management will provide written informed consent. Prior to the qualitative data collection, each participant will provide written informed consent. Participants will be anonymous in all publications. All data will be organised and handled with confidence and only the research team will have access to the data. Data will be stored according to the rules of The Danish Protection Agency.

Results will be disseminated continuously in the field of public health, for example, conference presentations, scientific articles and other platforms deemed relevant for the dissemination of this study.

Acknowledgements LSK would like to thank all the schools and teachers participating in the study. Also, a special thanks to Research and Innovation Centre for Human Movement and Learning, the University of Southern Denmark and the University College Lillebaelt.

Contributors LSK is principal investigator and initiated this paper and wrote the first draft. All authors contributed equally in deciding the overall study design, conducting and evaluating the pilot study, contributed in writing the manuscript and critically reviewing and discussing the content, approved the final version before submission and are equally responsible for all aspects of the study.

Funding The study is funded by The Faculty of Health at the University of Southern Denmark, the Department for the Study of Culture at the University of Southern Denmark and Research and Innovation Centre for Human Movement and Learning at the University of Southern Denmark and University College Lillebaelt.

Disclaimer The funders have no part in conducting the study, except approval of major adjustments of the research plan.

Competing interests None declared.

Patient consent Not required.

Ethics approval The Regional Committee on Health Research Ethics for Southern Denmark.

Provenance and peer review Not commissioned; externally peer reviewed.

Open Access This is an Open Access article distributed in accordance with the Creative Commons Attribution Non Commercial (CC BY-NC 4.0) license, which permits others to distribute, remix, adapt, build upon this work non-commercially, and license their derivative works on different terms, provided the original work is properly cited and the use is non-commercial. See: http://creativecommons.org/ licenses/by-nc/4.0/ (c) Article author(s) (or their employer(s) unless otherwise stated in the text of the article) 2018. All rights reserved. No commercial use is permitted unless otherwise expressly granted.

\section{REFERENCES}

1 Naylor PJ, Nettlefold L, Race D, et al. Implementation of school based physical activity interventions: a systematic review. Prev Med 2015;72:95-115.

2. Biddle SJ, Mutrie N, Gorely T. Psychology of physical activity: determinants, well-being and interventions. 3rd ed. Abingdon: Routledge, 2015.

3. Biddle SJ, Asare M. Physical activity and mental health in children and adolescents: a review of reviews. $\mathrm{Br} J$ Sports Med 2011;45:886-95.

4 Donnelly JE, Lambourne K. Classroom-based physical activity, cognition, and academic achievement. Prev Med 2011;52 Suppl 1:S36-42.

5. Chaddock L, Pontifex MB, Hillman $\mathrm{CH}$, et al. A review of the relation of aerobic fitness and physical activity to brain structure and function in children. J Int Neuropsychol Soc 2011;17:975-85.

6. Fedewa AL, Ahn S. The effects of physical activity and physical fitness on children's achievement and cognitive outcomes: a metaanalysis. Res Q Exerc Sport 2011;82:521-35.

7 Donnelly JE, Greene JL, Gibson CA, et al. Physical Activity Across the Curriculum (PAAC): a randomized controlled trial to promote physical activity and diminish overweight and obesity in elementary school children. Prev Med 2009;49:336-41.

8. Fedewa $\mathrm{AL}$, Ahn $\mathrm{S}$, Erwin $\mathrm{H}$, et al. A randomized controlled design investigating the effects of classroom-based physical activity on children's fluid intelligence and achievement. Sch Psychol Int 2015;36:135-53.

9. Government. Agreement between the Danish Government (the Social Democrats, the Social-Liberal Party and the Socialist People's Party), the Liberal Party of Denmark and the Danish People's Party on an improvement of standards in the Danish public school (primary and lower secondary education): Ministry of Education. 2013.

10. Jacobsen $\mathrm{RH}$, Bjørnholt B, Krassel KF, et al; En længere og mere varieret skoledag: Implementerings- og effektundersøgelse (A longer and more varied school day: Implementation and effect study. Copenhagen: The Danish Institute for Local and Regional Government Research, 2017.

11. Jacobsen RH, Flarup LH, Søndergaard NM. En længere og mere varieret skoledag: Kortlægningsrapport 2015 (A longer and more varied school day: Survey report 2015. Copenhagen: The Danish Institute for Local and Regional Government Research, 2015.

12. Strampel CM, Martin L, Johnson MJ, et al. Teacher perceived barriers and potential solutions to implementing daily physical activity in elementary schools. J Physic Health Educ 2014;80:14-22.

13. Dwyer JJ, Allison KR, Barrera M, et al. Teachers' perspective on barriers to implementing physical activity curriculum guidelines for school children in Toronto. Can J Public Health 2003:94:448-52.

14. Naylor PJ, Macdonald HM, Zebedee JA, et al. Lessons learned from Action Schools! BC-an 'active school' model to promote physical activity in elementary schools. J Sci Med Sport 2006;9:413-23.

15. Benes S, Finn KE, Sullivan EC, et al. Teachers' perceptions of using movement in the classroom. The Physical Educator 2016;73:110-35.

16. McMullen J, Kulinna P, Cothran D. Chapter 5 physical activity opportunities during the school day: classroom teachers' perceptions of using activity breaks in the classroom. J Physic Educ 2014;33:511-27.

17. Dinkel D, Schaffer C, Snyder K, et al. They just need to move: teachers' perception of classroom physical activity breaks. Teach Teach Educ 2017;63:186-95.

18. Stylianou M, Kulinna PH, Naiman T. Because there's nobody who can just sit for that long': teacher perceptions of classroom-based physical activity and related management issues. Eur Phy Educ Rev 2016;22:390-408.

19. Parks M, Solmon M, Lee A. Understanding classroom teachers perceptions of integrating physical activity: a collective efficacy perspective. J Res Child Educ 2007;21:316-28.

20. Cothran DJ, Kulinna PH, Garn AC. Classroom teachers and physical activity integration. Teach Teach Educ 2010;26:1381-8.

21. Day C, Smethem L. The effects of reform: have teachers really lost their sense of professionalism? J Educ Change 2009;10(2-3):141-57.

22 Day C. School reform and transitions in teacher professionalism and identity. Int J Educ Res 2002;37:677-92. 
23. Butler R. Teachers' achievement goal orientations and associations with teachers' help seeking: examination of a novel approach to teacher motivation. J Educ Psychol 2007;99:241-52.

24. Butler R. What teachers want to achieve and why it matters. In: Richardson PW, Karabenick SA, Watt HG, eds. Teacher Motivation Theory and Practice 1st ed. New York: Routledge, 2014.

25. Roth G, Assor A, Kanat-Maymon Y, et al. Autonomous motivation for teaching: how self-determined teaching may lead to self-determined learning. J Educ Psychol 2007;99:761-74.

26 Roth G. Antecedents and outcomes of teachers' autonomous motivation. In: Richardson PW, Karabenick SA, Watt HG, eds. Teacher Motivation Theory and Practice. 1st ed. New York: Routledge, 2014

27. SNd J, Lens W. An integrated model for the study of teachers motivation. Appl Psychol: An International Review 2005;54:119-34.

28. Goh TL, Hannon JC, Newton M, et al. "I'll squeeze it in": transforming preservice classroom teachers' perceptions toward movement integration in schools. Action in Teacher Education 2013;35:286-300.

29 Riley N Lubans D Holmes K, et al. Movement-based mathematics: enjoyment and engagement without compromising learning through the EASY minds program. EURASIA J Math Sci Tech Edu 2017;13:1653-73.

30. Deci EL, Ryan RM. The "what" and "why" of goal pursuits: human needs and the self-determination of behavior. Psychol Inq 2000;11:227-68.

31. Ryan RM, Deci EL. Self-Determination Theory and the facilitation of intrinsic motivation, social development, and well-being. Am Psychol 2000;55:68-78.

32. Fernet C, Senécal C, Guay F, et al. The Work Tasks Motivation Scale for Teachers (WTMST). J Career Assess 2008;16:256-79.

33. Deci EL, Ryan RM. Intrinsic motivation and self-determination in human behaviour. New York: Plenum Press, 1985.

34. Deci EL, Vallerand RJ, Pelletier LG, et al. Motivation and education: the self-determination perspective. Educ Psychol 1991;26(34):325-46.

35. Fernet C, Chanal J, Guay F. What fuels the fire: job- or task-specific motivation (or both)? On the hierarchical and multidimensional nature of teacher motivation in relation to job burnout. Work Stress 2017;31:145-63.

36. Jvd P, Volman M, Beishuizen J. Scaffolding in teacher-student interaction: a decade of research. Educational Psychology Review 2010;22:271-96.
37. Puntambekar S, Hubscher R. Tools for scaffolding students in a complex learning environment: what have we gained and what have we missed? Educ Psychol 2005;40:1-12.

38. Wood D, Bruner JS, Ross G. The role of tutoring in problem solving. J Child Psychol Psychiatry 1976;17:89-100.

39. Vygotsky LS. Mind in society - The development of higher psychological processes. Cambridge: Harvard University Press, 1978.

40. Tharp RG, Gallimore R. Rousing minds to life: teaching, learning and schooling in social context. Cambridge: Cambridge University Press, 1988.

41. Creswell JW, Clark VLP. Designing and conducting mixed methods research. 2nd ed. California: Sage Publications, Inc, 2011.

42. Tashakkori A, Teddlie C. SAGE handbook of mixed methods in social and behavioral research. 2nd ed. California: Thousand Oaks, 2010.

43. Ivankova NV, Creswell JW, Stick SL. Using mixed-methods sequential explanatory design: from theory to practice. Field methods 2006;18:3-20.

44. Morgan DL. Practical strategies for combining qualitative and quantitative methods: applications to health research. Qual Health Res 1998;8:362-76.

45. Teddlie C, Yu F. Mixed methods sampling: a typology with examples. J Mix Methods Res 2007;1:77-100.

46. Cha ES, Kim KH, Erlen JA. Translation of scales in cross-cultural research: issues and techniques. J Adv Nurs 2007;58:386-95.

47. McGorry SY. Measurement in a cross-cultural environment: survey translation issues. Qualitative Market Research: An International Journal 2000;3:74-81.

48. Brislin RW. Back-translation for cross-cultural research. J Cross Cult Psychol 1970;1:185-216.

49. Onwuegbuzie AJ, Combs JP. Emergent data analysis techniques in mixed methods research: a synthesis. In: Tashakkori A, Teddlie C, eds. SAGE handbook of mixed methods in social and behavioral research. 2nd ed. Thousand Oaks: SAGE Publications, 2017.

50. Creswell JW. Qualitative inquiry and research design: choosing among five approaches. 3rd ed. Thousand Oaks: SAGE Publications, 2013.

51. Kvale S. Doing nterviews. London: SAGE Publications, 2007.

52. Braun V, Clarke V. Using thematic analysis in psychology. Qual Res Psychol 2006;3:77-101.

53. Bazely P, Jackson K. Qualitative data analysis with NVIVO. 2nd ed. London: SAGE Publications, 2013. 